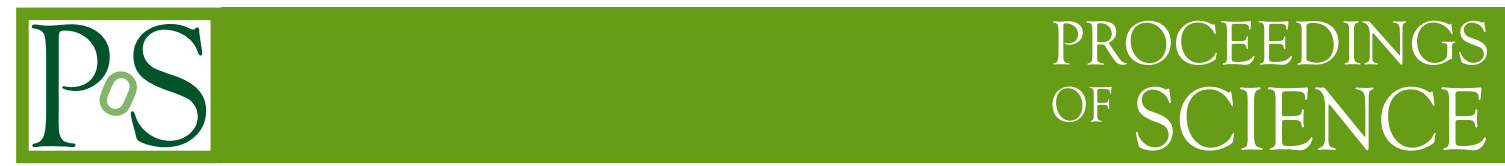

\title{
Heavy quarks as a key probe to the QGP properties
}

\author{
André Mischke ${ }^{* \dagger}$ \\ Institute for Subatomic Physics, Faculty of Science, Utrecht University, Princetonplein 5, \\ 3584 CC Utrecht, the Netherlands. \\ E-mail: a.mischkeduu.nl
}

In this contribution a selection of recent results on heavy-flavor production in high-energy heavyion collisions at the Relativistic Heavy Ion Collider (RHIC) at Brookhaven National Laboratory are reported. The total charm production cross section at mid-rapidity using various decay modes in different collision systems at $\sqrt{s_{\mathrm{NN}}}=200 \mathrm{GeV}$ exhibits a scaling behavior with the number of binary collisions. The energy loss of heavy quarks in the QCD medium is studied by the measurement of the heavy-flavor decay electron and $J / \psi$ yields in nuclear collisions.

8th Conference Quark Confinement and the Hadron Spectrum September 1-6 2008

Mainz, Germany

* Speaker.

${ }^{\dagger}$ A footnote may follow. 

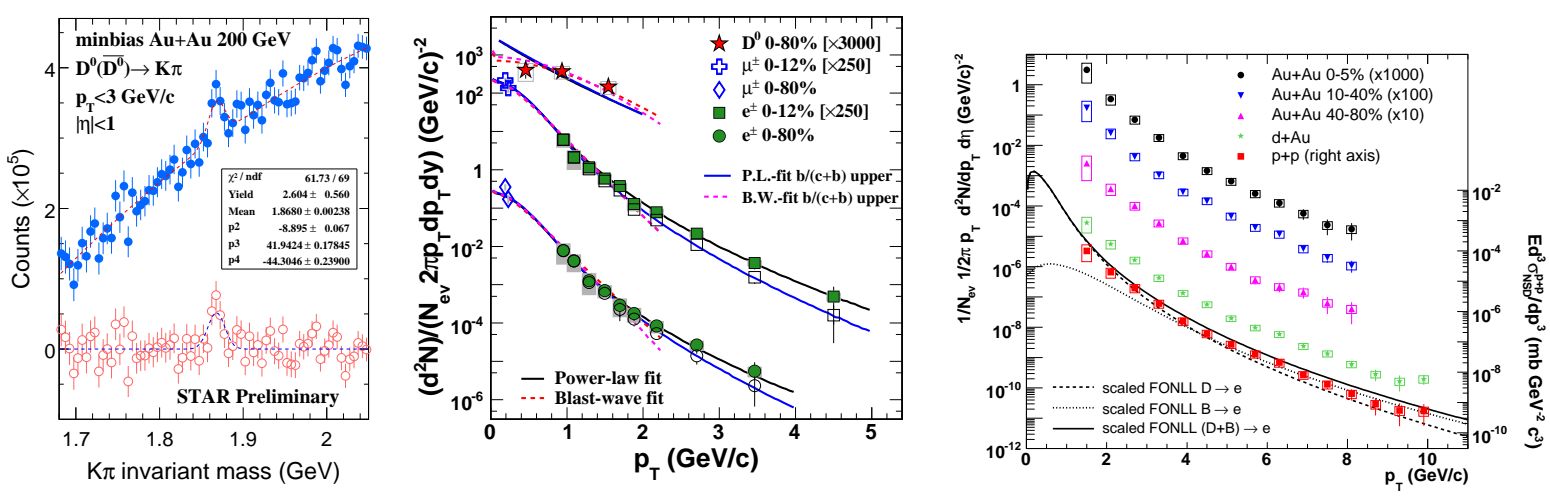

Figure 1: (Left panel) $\mathrm{K} \pi$ invariant mass distribution for $D^{0}+\overline{D^{0}}$ candidates before (blue circle) and after combinatoric background subtraction (red circle) in Au+Au minimum bias collisions at $\sqrt{s_{\mathrm{NN}}}=200 \mathrm{GeV}$. (Middle panel) Combined fit to extract charm production cross section using $D^{0}$, heavy-flavor decay $\mu$ and electron spectra before (solid squares and circles) and after (open squares and circles) bottom contribution subtraction. (Right panel) High $p_{\mathrm{T}}$ spectrum of heavy-flavor decay electrons from various collision systems.

\section{Introduction}

Quantum Chromodynamics (QCD), as the fundamental theory of strong interactions, predicts a phase transition from hadronic matter to a system of deconfined quarks and gluons, the Quark Gluon Plasma (QGP), if the surrounding temperature exceeds a critical value. The goal of highenergy heavy-ion physics is to produce such a deconfined QCD state and to study its properties under controlled laboratory conditions. Measurements at RHIC provide compelling evidences that the produced medium is indeed a plasma of quarks and gluons, but it behaves like a "perfect" fluid rather than an ideal gas [1]. One of the intriguing results is the measured jet attenuation owing to the energy loss of partons traversing through the hot, dense medium created in these reactions. The investigation of heavy-flavor production in such collisions allows verifying parton energy loss models and, therefore, offers important information about the properties of the produced QCD medium. Heavy quarks are expected to be produced predominantly in gluon fusion processes during the early stage of the collision. Theoretical models predict that heavy quarks should experience smaller energy loss than light quarks while propagating through the QCD medium due to the suppression of small angle gluon radiation (so-called dead-cone effect) [2, 3]. For a recent review see [4].

\section{Total charm production cross section}

The total charm cross section is determined from the measurements of open charm mesons and from the reconstruction of heavy flavor semi-leptonic decays via muon and electron measurements.

\subsection{Measurements of open charmed mesons, heavy-flavor decay $\mu$ and electrons in STAR}

The identification of the heavy-flavor decay products in the STAR experiment is performed using the Time Projection Chamber (TPC) and Time-of-Flight (ToF) $[5,6] . D^{0}$ mesons are reconstructed in the hadronic decay channel $D^{0} \rightarrow K^{-} \pi^{+}$by calculating the invariant mass of all 

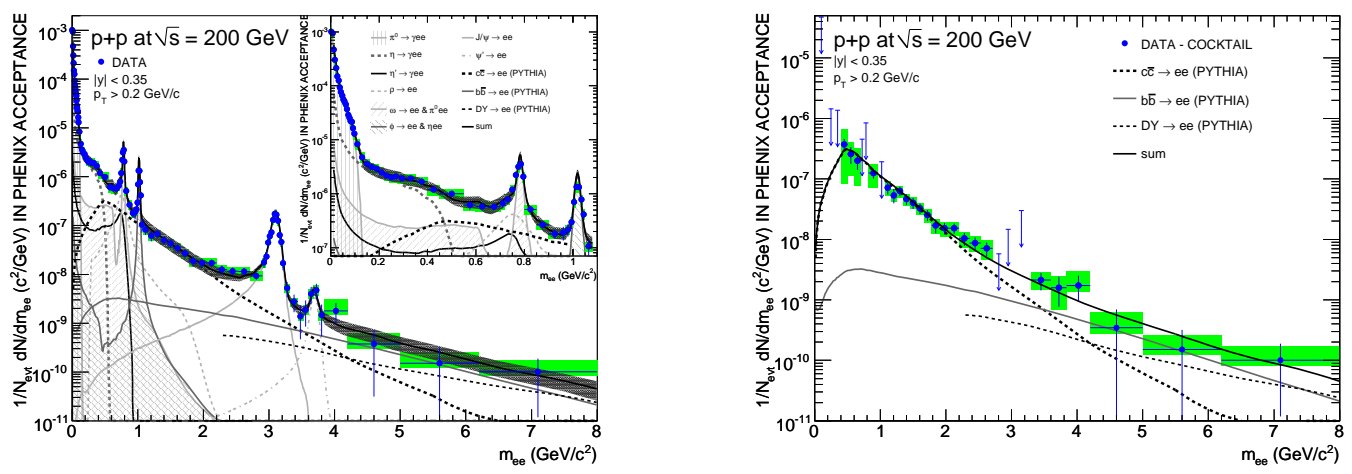

Figure 2: Di-electron mass spectrum in $p p$ collisions at $\sqrt{s_{\mathrm{NN}}}=200 \mathrm{GeV}$ before (left panel) and after (right panel) subraction of the hadronic background of known sources (normalized at $m_{e e}<20 \mathrm{MeV} / \mathrm{c}^{2}$ ). The curves in the right hand plot indicate different fits from the PYTHIA Monte-Carlo generator.

oppositely charged TPC particle track combinations (cf. Fig. 1 (left panel)). Muons at low $p$ $\left(p_{\mathrm{T}}<250 \mathrm{MeV} / c\right)$ are identified by the combination of the $m^{2}$ measurement in the ToF detector and the ionisation energy loss $(d E / d x)$ measurement in the TPC. A cut on the distance of closest approach is used to separate the prompt from decay muons. The yield of heavy-flavor decay electrons at low $p_{\mathrm{T}}$ is obtained by combining the $d E / d x$ and ToF measurements $(|1 / \beta-1|<0.03)$, whereas at high $p_{\mathrm{T}}$ the electron spectrum is obtained through measurements using TPC and the Electromagnetic calorimeter. The determination of the photonic electron background is based on invariant mass [7]. Figure 1 (middle panel) illustrates the $p_{\Gamma}$ spectra of $D^{0}, \mu$ and $e$ which cover $95 \%$ of the cross section. The charm cross section is found to be $\sigma_{\bar{c}}=1.40 \pm 0.11 \pm 0.39 \mathrm{mb}$ in the $12 \%$ most central $\mathrm{Au}+\mathrm{Au}$ collisions. The $p_{\Gamma}$ spectra for heavy-flavor decay electrons (so-called non-photonic electrons) is shown in Figure 1 (right panel) for different collision systems.

\subsection{Di-electron continuum measurement in PHENIX}

The PHENIX experiment has measured the electron-positron pair mass spectrum at midrapidity $(|\eta|<0.35)$ in $p p$ and $\mathrm{Au}+\mathrm{Au}$ collisions at $\sqrt{s_{\mathrm{NN}}}=200 \mathrm{GeV}[8,9]$. The electron identification is based on the Ring Imaging Cherenkov detector in conjunction with a highly granular calorimeter. The particle momentum is measured by Drift and Pad Chambers. Figure 2 (left panel) shows the measured $e^{+} e^{-}$pair yield per $p p$ collision with the so-called cocktail of known sources (hadronic background). The cocktail accounts for nearly the continuum in the mass region below $\sim 1 \mathrm{GeV} / c^{2}$. Except for the vector meson peaks, the $e^{+} e^{-}$pair in the mass range above $1.1 \mathrm{GeV} / c^{2}$ is dominated by heavy quarks correlated through flavor conservation. Figure 2 (right panel) depicts the $e^{+} e^{-}$pair yield after subtraction of the cocktail. The remaining components are expected to be from $c \bar{c}, b \bar{b}$ and Drell-Yan production processes. To get the charm cross section, the $\bar{e} e^{-}$pair yield is integrated in the range from 1.1 to $2.5 \mathrm{GeV} / \mathrm{c}^{2}$. The yield at full phase space is obtained by extrapolating the integrated yield to zero $e^{+} e^{-}$pair mass using PYTHIA Monte Carlo simulation. The rapidity distribution is taken from NLO pQCD calculation [10]. Contributions from $\bar{b}$ and Drell-Yan processes are estimated and properly subtracted. The total charm cross section is $\bar{\varphi}_{\bar{c}}=$ $544 \pm 39$ (stat.) \pm 142 (sys.) \pm 200 (model) $\mu b$. An alternative method is used to extract signals from $b \bar{b}$ production. The $e^{+} e^{-}$pair distribution after subtraction of the Drell-Yan contribution is fitted 

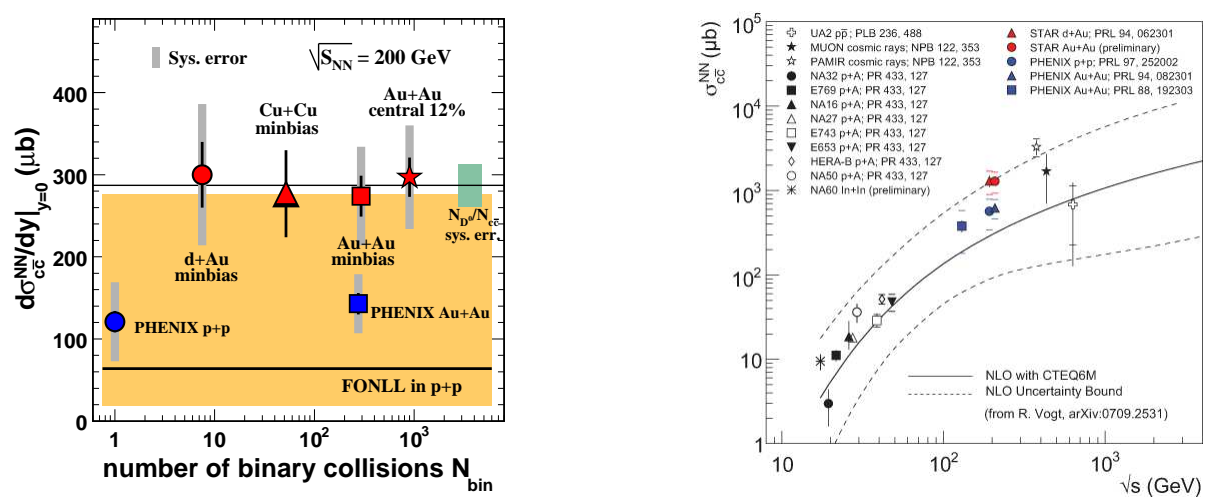

Figure 3: (Left panel) Total charm cross section at mid-rapidity per nulceon-nucleon collisions for different collision systems at $\sqrt{s_{\mathrm{NN}}}=200 \mathrm{GeV}$ as a function of the number of binary collisions $\left(N_{\text {bin }}\right)$. The FONLL calculation is shown as a band around the central value (thick solid line). (Right panel) Comparison of total charm cross section from STAR (red symbols) and PHENIX (blue symbols) with the world data. The dashed curves depict the uncertainty bands of the NLO pQCD calculations.

with the $e^{+} e^{-}$pair distributions from charm and bottom production generated with PYTHIA. The total bottom cross section is found to be $\sigma_{\bar{b} \bar{b}}=3.9 \pm 2.5(\text { stat })_{-2}^{+3}($ sys $) \mu b$. Both production cross sections are in agreement with previous results from non-photonic electron measurements [11].

In Figure 3 (left panel) the total charm cross section at mid-rapidity per nulceon-nucleon collisions for different collisions systems at $\sqrt{s_{\mathrm{NN}}}=200 \mathrm{GeV}$ is compared to FONLL calculations [12]. Within the large theoretical uncertainty, the measurements agree with the model calculations. The descrepancy between the STAR and PHENIX data is under investigation. The charm cross section follows binary collisons scaling, supporting the assumption that charm is predominantely produced by hard scattering processes in the initial state of the collision. The measured charm cross sections fit into the world data trend as shown in Fig. 3 (right panel). Also here, the large theoretical uncertainty leads to a little predictive power of the total charm cross section at higher collision energy.

\section{Energy loss of heavy quarks in the QCD medium}

Nuclear effects can be quantified by the nuclear modification factor $R_{A}$ where the particle yield in nuclear collisions is divided by the one in $p p$ interactions properly scaled by the number of binary collisions. $R_{A A}=1$ would indicate the absence of nuclear effects, and nucleus-nucleus collisions could be considered as a incoherent superposition of nucleon-nucleon interactions. The STAR and PHENIX measurements of $R_{A A}$ for non-photonic electrons at high $p_{\Gamma}$ in central $\mathrm{Au}+\mathrm{Au}$ collisions at $\sqrt{s_{\mathrm{NN}}}=200 \mathrm{GeV}$ are illustrated in Fig. 4 (left panel). The magnitude of $R_{\mathrm{AA}}$ is similar to the one observed for light-quark hadrons [7, 13], indicating an unexpected large energy loss of heavy quarks in the QCD medium, which is in contradiction to expections from the dead-cone effect. A comparison of the data to various theoretical model calculations shows that the observed suppression is overpredicted by the models using reasonalbe model parameters (see curves in Fig. 4 (left panel)). The data are described reasonably well if the bottom contribution to the non-photonic electrons is assumed to be small. However, azimuthal angular correlation measurements of non- 

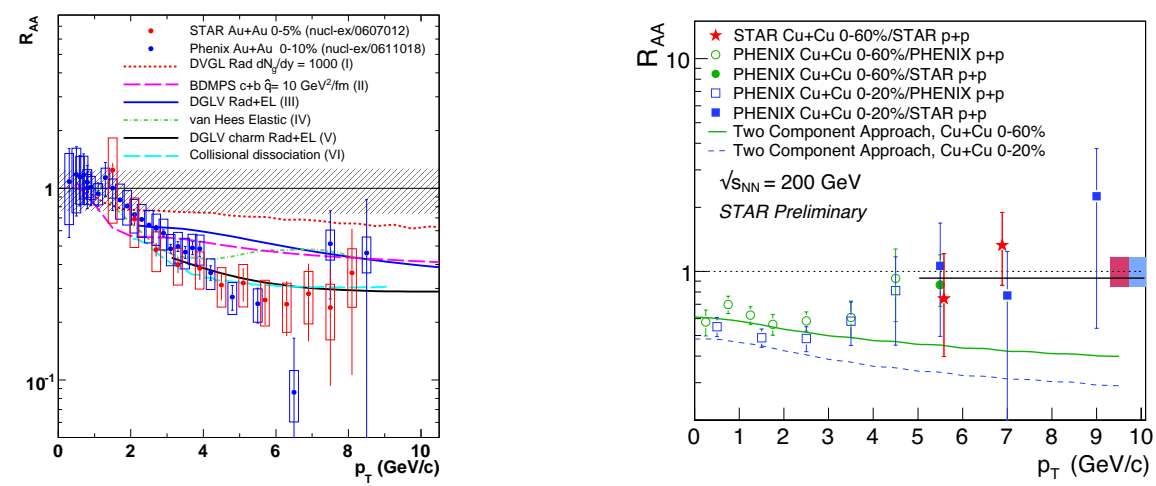

Figure 4: The nuclear modification factor $R_{A A}$ for heavy-flavor decay electrons in most central $\mathrm{Au}+\mathrm{Au}$ collisions (left panel) and for $J / \psi$ in $0-60 \% \mathrm{Cu}+\mathrm{Cu}$ collisions at $\sqrt{s_{\mathrm{NN}}}=200 \mathrm{GeV}$. The curves in the right hand plot are model predictions from the Two Component Approach.

photonic electrons and open charmed mesons in the STAR experiment have shown that the bottom decay yields are of comparable magnitude above $p r \sim 4 \mathrm{GeV} / c$ [15]. A possible scenario for $B$ meson suppression could be collisional dissociation in the medium [14].

The dissociation of quarkonia due to color-screening in a QGP is a classic signature of deconfinement in high-energy heavy-ion collisions $[16,17]$. PHENIX results have shown that the suppression of the $J / \psi$ as a function of centrality of the collision is similar to that observed at the SPS accelerator [19], even though the energy density reached in collisions at RHIC is significantly higher (factor 2-3). Theoretical prediction based on string theory application of AdS/CFT suggests that the effective $J / \psi$ dissociation temperature is expected to decrease with $\not 1$ [18]. This conjecture is different from the predictions of more traditional screening models where the suppression due to screening vanishes towards higher $p$. Recent data on the $R_{A A}$ for $J / \psi$ from the STAR [20] and PHENIX experiment [21] are shown in Fig. 4 (right panel). $R_{A A}$ is suppressed at low $p_{\mathrm{T}}$ (around $1 \mathrm{GeV} / c$ ), and the data suggest that $R_{A A}$ increases with $p_{\mathrm{T}}$ and reaches unity around $5 \mathrm{GeV} / c$, although the large errors currently preclude strong conclusions. However, this result is in contradiction with expectations from the Two Component Approach model [22], which predicts a suppression at high $p_{\mathrm{T}}$.

\section{Conclusions}

The total charm production cross section in high-energy heavy-ion collisions scales with the number of binary collisions, proving the assumption that charm is exclusively produced in the initial state of the collision. The observed strong suppression of heavy-flavor decay electrons at high $p_{\mathrm{T}}$ in central $\mathrm{Au}+\mathrm{Au}$ collisions together with the azimuthal angular correlation measurements in $p p$ collisions imply that $B$ production is stronger suppressed in nuclear collisions than expected. The $J / \psi$ nuclear modification factor $R_{A A}$ in $\mathrm{Cu}+\mathrm{Cu}$ collisions increases from low to high $p_{\Gamma}$ and reaches unity for $p_{\mathrm{T}}>5 \mathrm{GeV} / c$. This result is about $2 \sigma$ above the $R_{A A}$ at low $p_{\mathrm{T}}(<4 \mathrm{GeV} / c)$ and is consistent with no $J / \psi$ suppression. 


\section{Acknowledgments}

The author wish to thank the organizers for the stimulating atmosphere during the conference and especially for the wine taste reception. This work is supported by a fellowship of the Deutsche Forschungsgemeinschaft (DFG, MI 1143/3-1) and a Veni grant from the Netherlands Organisation for Scientific Research (project number 680-47-109).

\section{References}

[1] First three years of operation of RHIC, Nucl. Phys. A757, 1 (2005).

[2] Y.L. Dokshitzer and D.E. Kharzeev, Phys. Lett. B519, 199 (2001).

[3] M. Djordjevic, M. Gyulassy and S. Wicks, Phys. Rev. Lett. 94, 112301 (2005).

[4] A.D. Frawley, T. Ullrich and R. Vogt, Phy. Rep. 462, 125 (2008).

[5] J. Adams et al., Phys. Rev. Lett. 94, 062301 (2005).

[6] H. Zhang et al., Nucl. Phys. A774, 701 (2006).

[7] B.I. Abelev et al., Phys. Rev. Lett. 98, 192301 (2007).

[8] A. Adare et al., arXiv:0802.0050 (2008).

[9] A. Adare et al., arXiv:0706.3034 (2007).

[10] M.L. Mangano, P. Nason and G. Ridolfi, Nucl. Phys B405, 507 (1993).

[11] A. Adare et al., Phys. Rev. Lett. 97, 252002 (2006).

[12] R. Vogt, Eur. Phys. J. Special Topics 155, 213 (2008).

[13] A. Adare et al., Phys. Rev. Lett. 98, 172301 (2007). S.S. Adler et al., Phys. Rev. Lett. 96, 032301 (2006);

[14] A. Adil and I. Vitev, Phys. Lett. B649, 139 (2007).

[15] A. Mischke, Phys. Lett. B in press (arXiv:0807.1309). A. Mischke et al., J. Phys. G: Nucl. Part. Phys. 35, 104117 (2008).

[16] T. Matsui and H. Satz, Phys. Lett. B178, 416 (1986).

[17] F. Karsch, D. Kharzeev, and H. Satz, Phys. Lett. B637, 75 (2006) and arXiv:hep-ph/0512239 (2005).

[18] H. Liu, K. Rajagopal and U.A.Wiedemann, Phys. Rev. Lett. 98 (2007) 182301.

[19] A. Adare et al., Phys. Rev. Lett. 98, 232301 (2007).

[20] Z. Xu, T. Ullrich et al., arXiv:0809.2288 (2008).

[21] A. Adare et al., Phys. Rev. Lett. 101, 122301 (2008).

[22] X. Zhao and R. Rapp, Phys. Lett. B664, 253 (2008), private communication for $\mathrm{Cu}+\mathrm{Cu}$ predictions. 